\title{
Measures of symmetry
}

\author{
LEONARD ZUSNE \\ University of Tulsa, Tulsa, Oklahoma 74104
}

Three discrimination, rating, and ranking experiments were conducted to test the utility of two new single-form measures of symmetry in predicting response to plane figures. The predictive power of the new measures varied with the type of the perceptual task, prediction being better for tasks of a more clearly perceptual nature. The rest of variance in response was due to poorly defined conceptions of symmetry in many Ss, the confusion of symmetry with compactness, and the overestimation of symmetry in figures that approximate in appearance their ideally symmetric prototypes. These and previously conducted experiments lead to the conclusion that in many instances the symmetry parameter of random polygons has no significant influence on response variance and that, for figures of a given level of complexity, the only practically significant predictor of response is a measure of dispersion of shape contours.

In both common usage and in psychophysics of form, plane figures are most often conceived of as being either symmetric or asymmetric. At best, they are said to differ only in the number of axes of symmetry and are therefore richer or poorer in symmetry, depending on how many symmetry operations (reflection and rotation) can be performed on them without changing them. Symmetry, however, like other single-form parameters (complexity, dispersion, elongation, and linearity) is a continuum. Extensive experience with symmetric man-made objects and "geometric" figures in general is one of the reasons for the dichotomization of the continuum of symmetry and for the absence, until very recent times, of any continuous measures of symmetry in single forms.

In informational terms, symmetric forms are redundant because the information contained in one half of the form is repeated in the other half. Since redundancy is measurable in bits of information, so is symmetry, but here the problem arises of reconciling redundancy, which is a statistical concept, with symmetry of single shapes. Redundancy has to do with stimulus uncertainty, which cannot be specified except with reference to sets of stimuli. Redundancy, and therefore symmetry, as a statistical concept refers to the extent to which a stimulus sample is smaller than the total population that could exist. Garner (1970) suggests that when a shape is judged to be redundant it is not the shape itself that is so judged but the sample of shapes from which it is presumed to have come. Symmetric figures have few alternatives, and the very "best" figures, such as the circle or the square, are unique.

I have identified symmetry in single shapes with the third moment of areal and contour distribution in one-dimensional shape projections (Zusne, 1965). The third moment of area measures only a certain property of the distribution of the area of a shape in a projection. To refer to the third moment as a measure of symmetry of the untransformed figure, however, is not, strictly speaking, correct. It is the shape of the distribution of the one-dimensional projection whose redundancy is judged with respect to the size of the total population of such projections from which the one in question is presumed to have come.

Thus a translation between the statistical and determinate mathematical measures of symmetry is possible, and both kinds have been used in the past. The statistical measures have been used more often by far. Very fow measures of symmetry of the single form besides the third moment have been used. Brown, Hitchcock, and Michels (1962) defined a measure called areal asymmetry as the variance of the portions of the total area of a figure enclosed by each of the four quadrants when, in a given position of the figure, a horizontal and a vertical axis are passed through the geometric center of the figure. The relationship of this measure to symmetry is indeterminate since the position of the figure in which its areal asymmetry is measured varies randomly. When Ss are allowed to turn the stimulus shapes in all directions, areal asymmetry does not contribute anything to response variance in a scaling task (Zusne \& Michels, 1962b). Small's (1961) measure of horizontal, vertical, and quadrant balance did not turn out to be useful predictors in a similarity judgment task. The third moment of area and the third moment of the perimeter, along with the second and fourth moments, however, contributed significantly to response variance in a discrimination task in which six-choice oddity problems were exposed tachistoscopically (Zusne, 1965).

The computation of moments is so laborious that the use of the largest computers is mandatory. Of the configurational form parameters of dispersion, symmetry, and elongation (Zusne, 1970, Chap. 5), dispersion has proved itself to be by far the best predictor of performance in the work of many researchers (Zusne, 1970, Chap 6). While dispersion may be measured in terms of the second moment of area or of the perimeter, the simple ratio of the squared perimeter to the area enclosed by it works almost as well, provided comparisons are made between forms of the same level of complexity.

The experiments described below were performed, first of all, to provide a measure of symmetry of two-dimensional shapes comparable in simplicity to the $\mathrm{P}^{2} / \mathrm{A}$ measure and one that would be continuous rather than discrete. Another purpose was to compare the predictive power of any such new measure with that of the third moment measure and to establish its validity by giving the Ss several different perceptual tasks to perform. There was also a practical purpose. Whatever the limitations of random polygons, they are being used increasingly not only in the area of visual form perception but other, nonperceptual areas as well. It was thought that if a simple continuous measure of symmetry could be found, or if a need for such a measure could be shown not to exist, the work of researchers in these other areas would be facilitated.

\section{EXPERIMENT 1}

In this experiment an attempt was made to predict from a simple measure of symmetry, which was thought to be related to the third moment of area (U3A), performance in a discrimination task in which no constraints were imposed upon the S's information processing capacity. The rationale for the new measure was to replicate mechanically the process which presumably takes place when the degree of symmetry of a figure is evaluated, namely the actual or mental turning around of the figure to various positions until one is found that appears to maximize the degree of congruence of the two halves of the figure about some axis.

\section{Method}

Subjects. Ss were 46 students, 36 males and 10 females, enrolled in an introductory psychology course.

Apparatus and stimuli. A special device to measure symmetry (symmetrograph) was constructed. Variants of it have been used previously by Boynton, Elworth, Onley, and Klingberg (1960) and others. A vertically mounted $150-\mathrm{W}$ electric bulb 
illuminated the interior of a glass light-fixture globe through the $135-\mathrm{mm}$ fixture opening. The globe was $23 \mathrm{~cm}$ in diam and painted white inside. A silicon photocell was attached to the globe so that its axis was perpendicular to the direction of the light flux from the bulb. The average illumination of the inside surface of the globe was approximately proportionate to the total light flux entering it, so that the current output of the photocell varied directly with it. The $120-\mathrm{V}$ ac current of the power distribution system was stabilized with a Sola constant-voltage transformer. The output of the latter could be varied from 0 to $140 \mathrm{~V}$ by a $.63 \mathrm{KVA}$ Superior Electric Powerstat variable transformer. A horizontal Plexiglas table was mounted between the bulb and the globe opening. In contact with this table was a circular Plexiglas stage that was turned about its center by a small geared motor at a rate of $1.5 \mathrm{rpm}$.

A figure was cut in the middle of a sheet of opaque construction paper. Both the cutout and the hole were used. The cutout was mounted on a sheet of clear acetate and placed on top of the rotary stage so that its center of gravity coincided with the center of the stage and of the opening in the globe. The sheet of construction paper with the hole was turned upside down and centered in the same manner on top of the Plexiglas table, underneath the rotary stage. As the stage rotated, varying amounts of light entered the globe, depending on the amount of self-overlap in the figure in each successive position. The photocell output was increased by a two-transistor current amplification circuit and conducted to an Esterline Angus graphic ammeter, Model AW. The chart speed was set at 6 in. $/ \mathrm{min}$.

A recording was obtained in this manner from all 1008 -sided random polygons used in the moments study (Zusne, 1965). This relatively large sample of random polygons was chosen as the stimulus material to represent all levels of symmetry on a continuum. The recordings were examined, and for each polygon the point at which the symmetrograph output was lowest (maximum self-overlap) was noted.

Larger versions of these polygons were drawn in outline and offset-reproduced on white paper for Ss' use. Each sheet was cut to make a 16-sided regular polygon, in the center of which was located the stimulus figure.

Procedure. The experiment was conducted with the $46 \mathrm{Ss}$ as a group. Ss were seated so that they could not very well observe their neighbors' work. Each S was given a 12-in. unmarked wooden ruler and a pencil. The instructions were to draw a line through each figure so as to maximize the symmetry of the two halves of the figure. Examples of symmetric figures were shown and symmetry defined as the congruence of the two halves of a figure after reflection.

\section{Results}

Except for a few figures in which the placement of the lines was rather chaotic, in most figures they were drawn so that they passed through or close to the center of gravity. The lines were also examined for clustering. For each figure the Ss' responses were summarized by drawing a line that passed through the centroid of the figure and the middle of the cluster with the most lines. If there were two or three clusters in a figure with approximately equal numbers of lines in each of them, the most compact cluster was chosen.

Although the symmetrograms of most figures had from two to three troughs and the absolute values of many of them did not differ appreciably, the most stringent criterion was adopted, and disparities, in degrees, between the position of the perceptual axis of symmetry (PAS) and the position of maximum self-overlap (S-O) were measured.

Agreement between the judged and measured axes of symmetry was close: in 7 shapes the axes coincided exactly and in 67 shapes the disparity was only between 0 and $14 \mathrm{deg}$; in 30 shapes it ranged from 15 to $44 \mathrm{deg}$; and in only 3 cases was the disparity greater than $45 \mathrm{deg}$. The mean disparity was $15.7 \mathrm{deg}$. Since the maximum possible disparity was $90 \mathrm{deg}$, chance alone would have produced a mean disparity of about $45 \mathrm{deg}$. The clustering of the PAS about the measured symmetry maxima was thus statistically significant far beyond the .01 level (Student's $t$ ).

The predictive power of the U3A measure was also tested. The values of U3A had been previously computed for each of the 100 shapes and in each orientation 5 deg apart. The value of U3A about the PAS was then expressed as a percentage of the maximum value of $\mathrm{U} 3 \mathrm{~A}$ for a given shape. The mean deviation of the PAS from perfect symmetry in terms of U3A was $38.4 \%$, as compared to a $17.5 \%$ deviation from the point of maximum symmetry established by the symmetrograph.

\section{Discussion}

A substantial proportion of response variance could be predicted from the symmetrograms. Much of the remaining variance could also be explained, albeit in somewhat more phenomenological terms. It was observed that in many cases where Ss either consistently bypassed the center of gravity or drew the axis of symmetry in a position that did not coincide with the measured axis, such deviations were due to the attractiveness of some conspicuous landmark in the figure, such as a narrow, protruding angle. Ss would connect two such landmarks if they were in a more or less diametrical relationship, or else connect a conspicuous feature to the middle of a side opposite to it. Other "critical details" that clearly played a role in determining the drawing of the PAS were deep concave angles that seemingly divided a shape into two almost independent parts, three vertices pointing in the same direction, arrow- and rocket-shaped figures, shapes resembling the silhouette of a house, and the like. Bulky compact figures produced a dispersion of the lines drawn, but even here small protruberances, invaginations, and the like tended to concentrate some of the lines drawn.

A number of investigators have used the term "critical detail" without defining it exactly. The concept partly overlaps the concepts of "distinctive feature" and "physical form parameter." The relationship among these three concepts is probably the following. A critical detail can be pointed out exactly, but it has very limited generality. Distinctive features can be specified in dichotomous terms for small groups of objects, e.g., phonemes and graphemes (cf. Chap. 5 in Gibson, 1969). Distinctive features are common to several objects, but their number can be quite large nevertheless. They can be specified for polygons of 3,4 , and 5 sides, but beyond this point the procedure becomes unmanageable. Instead, a smaller number of parametric continuous measures of greater generality (e.g., moments) must be substituted. With increasing generality, however, comes a loss of predictive power for the single case. In addition, predictability from any one of these attributes of form is also a function of the perceptual task and of the stimulus sample used. Quantitative predictions along a continuum are possible if the task is suited for the utilization of parametric clues. With heterogeneous samples of stimuli, response to the more idiographic features of form may predominate in a subsample. Both processes were observable in Experiment 1.

\section{EXPERIMENT 2}

The maximum S-O measure developed in Experiment 1 was now tested in a symmetry rating task. It was hypothesized that if judgments of the degree of symmetry of a figure are made by evaluating the relative amount of overlap between two halves of it when one is mentally folded over the other, then the rating of symmetry should vary directly with the amount of self-overlap. 


\section{Method}

Subjects. Ss were 34 students, 20 males and 14 females, enrolled in an introductory psychology course.

Stimuli. The 1008 -sided random shapes used in Experiment 1 were cut from black construction paper and pasted on cardboard circles $15 \mathrm{~cm}$ in diam. All figures had the same longest extent but unequal areas. Equal-area shapes were folded about each of the axes indicated by troughs in the symmetrogram, as well as about the PAS, and the area of overlap was measured with a planimeter.

A sorting bin with seven compartments was constructed. The compartments were marked "least symmetric," 2, 3, 4, 5, 6, and "most symmetric."

Procedure. Ss were tested singly. Before the experiment proper, Ss were shown samples of figures differing in symmetry, dispersion of contours, and elongation, singly and in pairs, and asked to evaluate their symmetry. On the basis of this interview, seven potential $S$ s were discarded because they had never heard the term "symmetry" before, had a very vague or confused notion of it, or thought that it meant aesthetic appeal, elongation, and the like. Ss were then given the 100 stimuli and asked to rate them on a 7 -point scale by sorting them into the compartments of the sorting bin.

\section{Results}

Prior to computing the product-moment correlations reported below, appropriate scatterplots were prepared. None of the plots showed a curvilinear functional relationship that would require the computation of the eta coefficient.

Median ratings of symmetry ranged from 1.2 to 6.9 and their Qs from .3 to 2.0 . Pearson's $r$ between maximum $\mathrm{S}-\mathrm{O}$ and median symmetry ratings was .286 ( $<<.01)$; the correlation between S-O about the PAS and median ratings, however, was 333 . The correlation between dispersion (measured by the length of the perimeter since the area of all figures was the same) and median ratings was not statistically significant (.099). Correlations between maximum S-O and ratings and $\mathrm{S}-\mathrm{O}$ about the $\mathrm{PAS}$ and ratings were not statistically significant $(.270$ and .319), respectively).

Some 45 figures had one of the symmetrogram troughs just a few degrees removed from the PAS. S-O about this axis and rated symmetry correlated .432 $(p<.01)$. Correlation between ratings and self-overlap about the PAS in these figurys was also higher, $.385(\mathrm{p}<.01)$. Since bo.h predictors correlated .834 , the multiple correlation coefficient was only .435 $(p<.05)$, accounting for only $19 \%$ of response variance. Multiple regression analysis yielded regression coefficients that were not statistically significant.

In two previous studies (Zusne \& Michels, $1962 \mathrm{a}, \mathrm{b}$ ), it was found that when groups of figures, ranging from perfectly symmetric to very asymmetric ones, were rated on such properties as geometricity, regularity, and familiarity, Ss would first divide all figures into the symmetric and asymmetric subgroups, then rate the asymmetric subgroup on the basis of contour dispersion alone. It was noted that although the figures used in the present experiment were all asymmetric, a similar dichotomization had taken place: the 16 figures rated highest on symmetry (median ranks, 4.8 to 6.9 ) were symmetric in their own asymmetric way: these were figures which, if their imperfection were corrected, would be symmetric V-shapes, triangles, three-pointed "crowns," and 5-sided "house" shapes. Shapes rated lower did not resemble any such man-made objects or "geometric" figures. These 84 figures were therefore analyzed separately to see if the combined S-O and dispersion measures would predict symmetry ratings better in this truncated subsample of stimuli. Maximum S-O and ratings correlated $.277(p<.05)$ and length of the perimeter and ratings correlated .177 $(p>.05)$. The correlations of ratings with $\mathrm{S}-\mathrm{O}$ and perimeter for the 16 figures rated most symmetric did not reach the .05 level of significance.

The form of the symmetrograms could not be related in any way to symmetry except in terms of the troughs that indicated positions of greatest self-overlap. While bulky shapes (with most angles convex) produced a relatively flat curve and were rated, in general, toward the symmetric end of the scale, the figure rated most symmetric produced a curve with the most pronounced peaks and troughs. Flatness or peakedness of the symmetrograms is primarily related to elongation, and the polygon rated most symmetric had a thin, V-like form.

\section{Discussion}

In contrast to Experiment 1, in this experiment the S-O measure was not nearly as good a predictor of performance. Even though some of the correlations were statistically significant beyond the .01 level, the absolute size of the correlations was such that only a small percentage of total response variance could be predicted from the S-O measure. The different nature of the perceptual task used in this experiment is one possible explanation of this result. The axis-drawing task is a task more directly related to the comparison of areas about an axis. It does not force the $S$ to compare the magnitude of self-overlap of one shape with that of another or with any mentaily held standard. When the rating task does force such comparisons, additional factors, unrelated to the independent variable, begin to affect response, such as the assimilation of shapes that only look symmetric to ideally symmetric prototypes of these shapes. As a result, they are rated more symmetric than the independent variable would predict. For instance, the figure rated most symmetric (median rank 6.9, Q $=.3$ ) looked like an imprecise, free-hand drawing of a chevron. The PAS and the measured point of maximum $\mathrm{S}-\mathrm{O}$ coincided perfectly. Self-overlap between the two branches of the figure was relatively high, but it was less than in quite a number of other figures rated much. lower on symmetry.

\section{EXPERIMENT 3}

In this experiment, the U3A, S-O, and a new symmetry measure were tested in a ranking task in which symmetry was left undefined. The purpose of the experiment was to see what effect the lack of a definition of symmetry would have on the utilization of physical form dimensions in a symmetry judging task. Unless the researcher is directly concerned with symmetry, it is not likely that symmetry will be defined for the $S$ in any task in which random shapes are employed. It was reasoned that if physical form dimensions do not predict symmetry judgments in a task where the S's attention is called to symmetry or asymmetry, then his judgments would be even less influenced by such form attributes when no reference is made to them. The practical consequence would be that symmetry measures need not be considered with shapes that are clearly asymmetric.

\section{Method}

Subjects. Ss were 24 students, 16 males and 8 females, enrolled in an introductory psychology course.

Stimuli. A group of 16 polygons covering the entire range of values of $\mathrm{U} 3 \mathrm{~A}$ and approximately equally spaced on that measure (Group A) were selected from the 100 8-sided random polygons used in Experiments 1 and 2 . An additional measure, called asymmetry of coordinates (AC), was computed on the 100 shapes as follows. A shape was placed in a coordinate system so that its center of gravity coincided with the origin and the PAS coincided with the $y$ axis. The $x$ coordinates of the vertices in Quadrants II and III were measured (algebraic sign ignored) and their sum subtracted from the sum of $x$ coordinates in Quadrants $I$ and 
IV. Likewise, the sum of y coordinates in Quadrants II and III was subtracted from the sum of $y$ coordinates in Quadrants I and IV. Symmetry was defined as the sum of the two remnants: the more symmetric the figure, the smaller the sum. From the 100 shapes, 16 representing the range of values of $\mathrm{AC}$ and spaced about equally throughout the range were selected to form a second group of stimuli (Group B). Since 5 shapes were common to both groups, a total of 27 different shapes were used. The shapes were reproduced in outline on $8 \frac{1}{2} \times 11$ in. index card stock. A small arrow at one side of the shape indicated the "up" position. This position corresponded to the placement of the shape in the coordinate system for the purpose of computing AC.

Procedure. Ss were tested singly and in groups of two and three. One-half of the Ss were given Group A of stimuli first and Group B second. With the other half of Ss the sequence was reversed. The instructions were to rank the 16 figures on symmetry in the "up" position indicated by the arrow. Ss were told to use whatever idea of symmetry they had. There was enough space for $S s$ to spread out the 16 sheets and to look at all figures at approximately the same time. Having arranged the figures according to symmetry, Ss turned the sheets face down and wrote the ranks (1 through 16) on the backs of the sheets.

\section{Results}

Intercorrelations (Spearman's rank-order correlation coefficient) were computed between the $A C, S-3$, and length of perimeter measures and median symmetry ranks. This was done separately for the two groups of stimuli. None of the correlations was statistically significant $(\mathrm{p}>.05)$. Ranking done by one-half of the $\mathrm{Ss}$ correlated with that of the other half .68 and .94 for Groups A and B of stimuli, respectively.

Scatterplots were prepared for each $S$. None of the Ss appeared to have used U3A in their judgments. Of the $24 \mathrm{Ss}, 10$ showed low to moderate correlation between ranking and $\mathrm{AC}$; eight of the relationships were positive and two negative. Twenty-three of the 24 Ss showed some relationship between ranking and dispersion of contours. Variability in the use of this parameter was the rule. A rectilinear relationship, positive or negative, was observed in some Ss, ranging from low to high in magnitude. Some Ss showed an inverted-U relationship, i.e., judging both the most compact and the least compact shapes as symmetric. In addition, 10 Ss showed changes in the use of physical clues from the first group of shapes to the second. The changes were either from no use of any predictor to the use of one of them, in the algebraic sign of the correlation, or from a curvilinear relationship to a rectilinear one.

As in Experiment 2, here also the overriding effect of assimilation toward symmetry prototypes determined by past experience was observed: pronounced halving of shapes by deep invaginations, arrow- or rocket-shaped figures, and the like were judged to be more symmetric than others, without regard to any other physical properties.

\section{Discussion}

Under conditions of undefined symmetry the only identifiable clue utilized by $\mathrm{Ss}$ was the dispersion of contours. There was no consistency in the manner in which it was used, however, which resulted in an overall nonsignificant correlation between it and symmetry ratings. The configurational clues reflected in the specific measures of symmetry, simple (AC, S-O) or complex (U3A), were either not used at all or only by a few Ss. Thus, this experiment extends the findings of two previous ones (Zusne \& Michels, $1962 \mathrm{a}, \mathrm{b})$, in which both symmetric and asymmetric 4- and 5-sided polygons were rated on geometricity, regularity, and familiarity, In these experiments, Ss rated all symmetric shapes as being the most geometric, regular, or familiar regardless of the particular term used in the instructions. Within the asymmetric subsample, figures were then rated as more or less geometric, etc., according to their perceived compactness. Such was also the finding in the present experiment, except that figures rated most symmetric were ones resembling well-known symmetric objects and geometric forms.

\section{CONCLUSIONS}

(1) Except for the dichotomous division of plane figures into the perfectly symmetric and other, asymmetric figures, most individuals have a poorly developed concept of symmetry, to the point where it may be completely absent. The term "symmetry" is apt to be treated on a par with such terms as "regularity," "familiarity," and "geometricity," although the overlap among these concepts is only partial. The ordering of asymmetric plane figures along continua designated by these terms is done mostly on the basis of contour dispersion (compactness).

(2) Ss respond or fail to respond to measured physical properties of figures that reflect symmetry as a function of the nature of the perceptual task given them. In ranking, rating, and scaling tasks where the $S$ is required to compare, evaluate, or judge each stimulus in terms of a verbal label, defined for him or not, response cannot be predicted from such measures. In tasks that are more perceptual and involve less symbolic activity, such as discrimination under tachistoscopic conditions or the physical bipartition of figures, performance can be predicted from physical form measures at statistically and practically significant levels.

(3) As a practical consequence, in a sample of random shapes symmetry may be either considered or ignored depending on the nature of the task and the purpose that the researcher seeks to accomplish. In many instances, the only form parameter, within a given complexity level of the form, that needs to be measured to predict response is dispersion of the contours.

\section{REFERENCES}

BOYNTON, R. M., ELWORTH, C. L., ONLEY, J. W., \& KLINGBERG, C. L. Form discrimination as predicted by overlap and area. RADC-TR-60-158, September 1960.

BROWN, D. R., HITCHCOCK, L., JR., \& MICHELS, K. M. Quantitative studies in form perception: An evaluation of the role of selected stimulus parameters in the visual discrimination performance of human subjects. Perceptual \& Motor Skills, 1962, 14, 519-529.

GARNER, W. R. Good patterns have few alternatives. American Scientist, 1970, 58, 34-42.

GIBSON, E. J. Principles of perceptual learning and development. New York: Appleton, 1969.

SMALL, V. H. Judged similarity of visual forms as functions of selected stimulus dimensions. Unpublished doctoral dissertation, Purdue University, 1961. Dissertation Abstracts, 1962. $22,2481-2482$.

ZUSNE, L. Moments of area and of the perimeter of visual form as predictors of discrimination performance. Journal of Experimental Psychology, 1965, 69, 213-220.

ZUSNE, L. Visual perception of form. New York: Academic Press, 1970.

ZUSNE, L., \& MICHELS, K. M. Geometricity of visual form. Perceptual \& Motor Skills, 1962a $14,147-154$

ZUSNE, L., \& MICHELS, K. M. More on the geometricity of visual form. Perceptual \& Motor Skills, 1962b, 15, 55-58.

(Accepted for publication October 1, 1970.) 